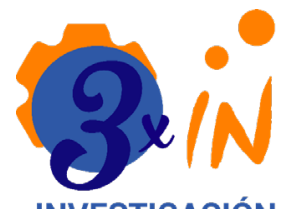

INVESTIGACIÓN E INNOVACION en INGENERIAS ISSN2344-8652

Recibido: 10/06/2021 Aceptado: 27/08/2021 Publicado: 13/09/2021

Correspondencia de autores: johernandez79@uan.edu.co

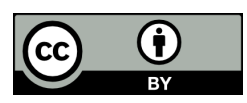

Copyrith 2020 by Investigación e Innovación en Ingenierías

\section{Modelo multicriterio AHP-TOPSIS: una estrategia para evaluar alternativas de generación de energía eléctrica a partir de fuentes renovables}

\section{AHP-TOPSIS multi-criteria model: a strategy to evaluate alternatives for the generation of electricity from renewable resources}

\author{
José Daniel Hernández Vásqueza ID Jhonathan Eduardo Solano De La Rosa iD \\ Farid Enrique Ferreira Medina
}

Universidad Antonio Nariño, Colombia

Resumen

Objetivo: Evaluar el desempeño de tecnologías para la generación de energía eléctrica a partir de fuentes renovables en la costa caribe colombiana utilizando el modelo multicriterio AHP-TOPSIS. Metodología: La motivación para el desarrollo de este tema se fundamenta en la necesidad identificada por el sector energético de la región para identificar alternativas que permitan suplir las necesidades del consumo de energía regional, además de contribuir a una matriz energética nacional sostenible. Considerando que una determinada tecnología puede ser más viable, en base a la información disponible en la literatura especializada, el problema fue analizado teniendo en cuenta tres criterios: económico, ambiental y técnico. La metodología analítica adoptada se basa en la aplicación de métodos multicriterios híbridos AHP-TOPSIS. Resultados: Los resultados consolidados permitieron: (i) establecer un ranking de las distintas tecnologías para la generación de energía eléctrica a partir de fuentes renovables, siendo la energía Fotovoltaica aquella de mayor peso y la Energía Hidráulica la de menor peso; (ii) confirmar que el criterio económico es el más crítico al momento de evaluar el desempeño de una tecnología. De igual forma, se estableció que el riesgo de la inversión es el subcriterio más crítico de los nueve evaluados. Conclusiones: El trabajo permitió concluir que el dinamismo del mercado internacional, diversas políticas sustentables, así como la actual crisis política que repercute en los países latinoamericanos permiten explicar la inestabilidad de la economía en pro de invertir en alternativas tecnológicas. Razón por la cual, el criterio económico y el subcriterio relacionado al riesgo de la inversión fueron considerados los más críticos en el estudio realizado.

Palabras clave: Energías limpias, Fuentes de energía renovables, Generación de energía eléctrica, Matriz energética, Método multicriterio AHP-TOPSIS.

Abstract

Objective: ETo evaluate the performance of technologies for the generation of electricity from renewable sources in the Colombian Caribbean Coast using the AHP-TOPSIS multi-criteria model. Methodology: The motivation for the development of this issue is based on the need identified by the energy sector in the region to identify alternatives that allow meeting the needs of regional energy consumption, in addition to contributing to a sustainable national energy matrix. Considering that a certain technology may be more viable, based on the information available in the specialized literature, the problem was analyzed taking into account three criteria: economic, environmental and technical. The analytical methodology adopted is based on the application of hybrid multicriteria AHP-TOPSIS methods. Results: The consolidated results allowed: (i) to establish a ranking of the different technologies for the generation of electrical energy from renewable resources, with Photovoltaic Energy being the one with the greatest weight and Hydraulic Energy the one with the least weight; (ii) confirm that the economic criterion is the most critical when evaluating the performance of a technology. Similarly, it was established that the investment risk is the most critical sub-criterion of the nine evaluated. Conclusions: The work allowed to conclude that the dynamism of the international market, various sustainable policies, as well as the current political crisis that affects Latin American countries allow to explain the instability of the economy in favor of investing in technological alternatives. Therefore, the economic criteria and the sub-criteria related to investment risk were considered the most critical in the study carried out.

Keywords: AHP-TOPSIS multi-criteria method, Clean energies, Electricity generation, Energy matrix, Renewable energy sources.

Como citar (IEEE): J. Hernández-Vásquez., J. Solano De La Rosa y F. Ferreira-Medina "Modelo multicriterio AHP-TOPSIS: una estrategia para evaluar alternativas de generación de energía eléctrica a partir de fuentes renovables", Investigación e Innovación en Ingenierías, vol. 9, n², 175-191, 2021. DOI: https://doi.org/10.17081/invinno.9.2.4482 


\section{Introducción}

La producción de energía eléctrica en Colombia es esencialmente hidráulica, obteniendo uno de sus picos más altos de generación en 2018 con un 82,16\% [1]. Sin embargo, la generación de energía eléctrica se ha venido realizando a partir de fuentes naturales renovables en distintos países del mundo [2,3,4], lo que se ha tornado en una actividad importante para la conservación del medio ambiente y la protección de las futuras generaciones. A nivel nacional, a partir de los objetivos perseguidos por el Plan Nacional de Desarrollo y la existencia del Sistema Interconectado Nacional, la inclusión de fuentes renovables en la matriz energética es una estrategia fundamental para el futuro energético a mediano plazo del país, así como la búsqueda continua de cumplir con las políticas internacionales de sostenibilidad [5,6,7]. Cabe destacar la importancia de profundizar en el análisis de las distintas fuentes de energía en la Costa Caribe y las tecnologías requeridas para tal fin. Por ejemplo, la energía presente en el movimiento natural del océano, la energía fotovoltaica, energía termosolar, energía eólica proveniente de las corrientes de viento del océano, entre otros tipos. Madrid y Toro [8] afirman que en Colombia el aprovechamiento de recursos hídricos a mediana y pequeña escala ha sido muy reducido, es decir aprovechamientos del orden de $1 \mathrm{~kW}$ hasta $500 \mathrm{~kW}$. Además, indican que uno de los métodos más usados para generar energía eléctrica, es mediante la inducción electromagnética.

El principio radica en transformar la energía cinética en energía eléctrica mediante un generador. Por otra parte, Isaza [9] indica que el aprovechamiento de energía renovable con la condición financiera más favorable es la energía eólica. El autor afirma que la energía solar fotovoltaica, cuenta con altos costos de inversión, además que las energías hidrocinética y mareomotriz requieren precios sostenidos de mercado. Beltrán [10] explica una de las medidas del gobierno nacional para incentivar la generación de energía eléctrica con tecnologías no convencionales con el objetivo de atenuar la generación de gases de efecto invernadero, atender la demanda de energía eléctrica en Colombia y consolidar nuevas conexiones al sistema interconectado nacional. Por los cual se creó la ley 1715 de mayo de 2014, la cual ofrece beneficios como la exención de impuestos de valor agregado para proyectos de energía eléctrica con fuentes renovables en sus etapas de diseño, ejecución, puesta en servicio, materiales y equipos. Además, se brinda una reducción del $50 \%$ en la declaración de renta de la inversión realizada en el respectivo proyecto entre otros beneficios.

En comparación con los países de la comunidad andina, un estudio realizado por Castro et al. [11] mostró un análisis que utiliza una serie histórica de datos que van desde 2000 a 2015, en función de la información disponible de las agencias oficiales de energía. Como resultados principales, se muestran tendencias de indicadores similares entre los países miembros: Colombia, Ecuador y Perú, que conforman un grupo, mientras que Bolivia muestra indicadores distantes de este grupo. El estudio, en general, confirma que el grupo de países tiene muchas oportunidades para lograr avances significativos en los temas de energía económica y alcanzar altos niveles de desarrollo. Con relación a las regiones geográficas de Colombia, Maldonado et al. [6] evaluaron el potencial solar del Departamento Santander por medio de una distribución nodal a través de la construcción de una malla. El trabajo permitió establecer los municipios con mayor potencial, además de calcular los factores que determinan el rendimiento de un sistema solar fotovoltaico. En 2018, Corredor [12] analizó la situación de la matriz energética colombiana y las políticas del país de cara a un proceso de transición hacia energías limpias. En esta misma línea de investigación y, en el mismo año 2018, Montes [13] afirmó que el calentamiento global, producto principalmente de la quema de carbón, petróleo y gas, podría llevar a Colombia a una situación en la que, paradójicamente, la única alternativa para que el país tenga independencia y seguridad energética sea el carbón. Aún en 2018, Romero et al. [14], considerando el punto de vista social, evaluaron las implicaciones distributivas de un impuesto a las 
emisiones de dióxido de carbono sobre el bienestar de los hogares, a través de cambios en los precios de la canasta consumida. Como conclusión del estudio, los autores presentan una serie de recomendaciones de política para lograr los efectos deseados, tanto en reducción de emisiones como en la incorporación de criterios de bienestar en las medidas de mitigación. Pensado en el mediano y largo plazo, Moreno et al. [15] aplicaron una metodología de escenarios para caracterizar las alternativas energéticas basada en premisas vigentes y en perspectivas de desarrollo. Los resultados muestran una disminución de 6000 GWh a 2030 obtenidos por la aplicación de planes de eficiencia energética y un nivel de integración de las Fuentes No Convencionales de Energía (FNCE) de aproximadamente un 20\%. En relación con la aplicación de métodos multicriterio, Ocampo et al. [16] utilizaron el método AHP para estudiar la gestión del Riesgo en la Implementación de Sistemas Fotovoltaicos. Los autores establecieron que, de los criterios técnicos, el que tiene mayor peso es el riesgo diseño, seguido del riesgo operacional y del riesgo técnico. De los criterios económicos, el que tiene mayor peso es el riesgo costos, seguido de los riesgos financieros y de los riesgos externos. En esta misma tecnología, Romero et al. [17] plantearon un análisis acerca del impacto del marco normativo/regulatorio vigente del sector eléctrico colombiano referente a la inclusión de energías renovables al sistema de distribución eléctrico, en especial los sistemas fotovoltaicos (SFV).

Los autores afirman que con la inclusión de las FNCE se logra ampliar la matriz energética, se empieza a cumplir los compromisos adquiridos en reducir las emisiones de gases de efecto de invernadero, se logra robustez al sistema eléctrico y se garantiza el suministro en tiempos de sequía. En relación con las fuentes alternativas, un estudio realizado por Porras et al. [18] evidencia que la falta de suministro afecta los indicadores de competitividad de un país o región, lo cual no permite el desarrollo adecuado de tecnologías de energía no convencional tales como solar, biomasa y eólica. En este sentido, Rojas et al. [19] proponen una inversión a la conversión del parque automotor hacia el gas natural consiguiendo favorablemente la reducción de enfermos por vías respiratorias, de costo por salud para el estado, y reducción de la contaminación atmosférica. Finalmente, Medina et al. [20] presentan un panorama de la actualidad colombiana en las energías renovables resaltando aquellos proyectos que se destacan a nivel nacional que aprovechan las fuentes de energías renovables presentes en el territorio nacional, mostrando información de primera mano sobre la evolución del mercado de las energías renovables en América Latina y los beneficios que trae consigo su aplicación para aquellos países que optan por ellas.

Considerando el panorama actual, este trabajo fundamenta su motivación por los desafíos energéticos que atraviesa la región caribe colombiana y la necesidad de conocer los aspectos (criterios/subcriterios) más importantes que influyen en la aplicación de tecnologías para la generación de energía eléctrica a partir de fuentes renovables en la costa caribe colombiana. De esta forma, el trabajo busca evaluar el desempeño de tecnologías para la generación de energía eléctrica a partir de fuentes renovables en la costa caribe colombiana utilizando el modelo multicriterio AHP-TOPSIS.

\section{Metodología Aplicada}

Este trabajo basó su metodología en la aplicación de modelos multicriterio, más específicamente, el modelo hibrido AHP-TOPSIS. En primer lugar, se definieron los criterios y subcriterios en base a una extensa revisión de la literatura especializada. Esta etapa se constituye en una de las más importantes y críticas de la investigación. Es conocido que son múltiples los factores y/o criterios que influyen en una determinada fuente para la generación de energía. En ese sentido, las distintitas tecnologías para la generación de energía eléctrica serán evaluadas a partir de diversos aspectos: criterios y subcriterios. Por ejemplo: ambiental, político, social, técnico, económico, entre otros. Estos criterios fueron obtenidos con el objetivo 
de estar en sintonía al estado del arte y que la selección de los criterios/subcriterios estén alineados a aquellos del contexto nacional e internacional. De esta forma, el estudio bibliográfico y el conocimiento especializado del contexto regional permitieron una definición adecuada de los distintos criterios en los cuales se enmarcó la investigación. Posteriormente, se procedió a realizar el diseño y aplicación de un instrumento de investigación, es decir, un formulario con preguntas y respuestas con opción múltiple, que fue desarrollado con la herramienta web de Google Form [21]. El objetivo de este instrumento es conocer la opinión de un notable grupo de especialistas del área (industria y academia), previamente seleccionado, sobre las mejores alternativas tecnológicas para la generación de energía eléctrica a partir de fuentes renovables en la costa caribe colombiana. Esta etapa es fundamental, una vez que los resultados de la investigación tendrán su sustento en la opinión de este selecto grupo de especialistas. El método multicriterio de apoyo a la decisión seleccionado (AHP-TOPSIS) fue aplicado a los resultados arrojados por el instrumento de investigación. A manera de ejemplo, se explicará, brevemente, la aplicación de esta etapa: cada especialista recibió un link para la realización del instrumento de investigación. El especialista, considerando su conocimiento y experiencia en el área, debió escoger entre dos alternativas tecnológicas cual es la de su preferencia (ejemplo: seleccionar una opción entre la energía solar y la energía eólica, para generar energía eléctrica).

Este primer análisis, permitió definir cuál fuente de energía es la más adecuada bajo una comparación directa par a par. Seguidamente, se realizó una comparación entre cada alternativa, pero en este caso, dicha comparación estuvo en función de un determinado criterio con el fin de determinar el criterio más crítico (ejemplo: considerando el criterio económico, cuál de las siguientes dos alternativas es la más adecuada, energía solar o energía eólica.). Finalmente, se realizó una evaluación de los subcriterios para definir cuál es el más crítico, mediante una comparación par a par (ejemplo: considerando el criterio económico, cuál de los siguientes subcriterios es el de su preferencia, costo de la inversión inicial o costo de operación/ mantenimiento de la tecnología). Cada especialista tuvo un espacio para agregar algún comentario relevante para sustentar su selección. Finalmente, una vez obtenidos los resultados consolidados en el instrumento de investigación, se procedió a realizar un análisis detallado de los mismos, mediante la aplicación del método multicriterio hibrido AHP-TOPSIS, con el propósito de discutir los aspectos más relevantes obtenidos y proponer estrategias que permitan avanzar en el conocimiento y eficiencia de la matriz energética nacional.

\section{Método Ahp-Topsis}

El método AHP (Analytic Hierarchy Process) es una herramienta de toma de decisiones ampliamente utilizada para determinar prioridades entre diferentes criterios, subcriterios y alternativas. Fue introducido y definido por Thomas Saaty en 1977 [22] como una herramienta eficaz para tratar la toma de decisiones complejas y que puede ayudar al tomador de decisiones a establecer prioridades y tomar la mejor decisión.

En términos generales, el modelo AHP se puede dividir en tres etapas: (i) descomposición, (ii) juicios comparativos y (iii) síntesis de prioridades [23,24]. En primer lugar, la descomposición consiste en estructurar el problema a nivel jerárquico, donde el objetivo ocupa el primer nivel, los atributos ocupan niveles intermedios y el último nivel presenta las alternativas a juzgar. La Figura 1 ilustra una estructura jerárquica genérica con cuatro niveles. En esta figura, se puede observar que el objetivo está relacionado con alternativas a través de criterios y subcriterios [22]. 
Figura 1 - Estructura jerárquica genérica

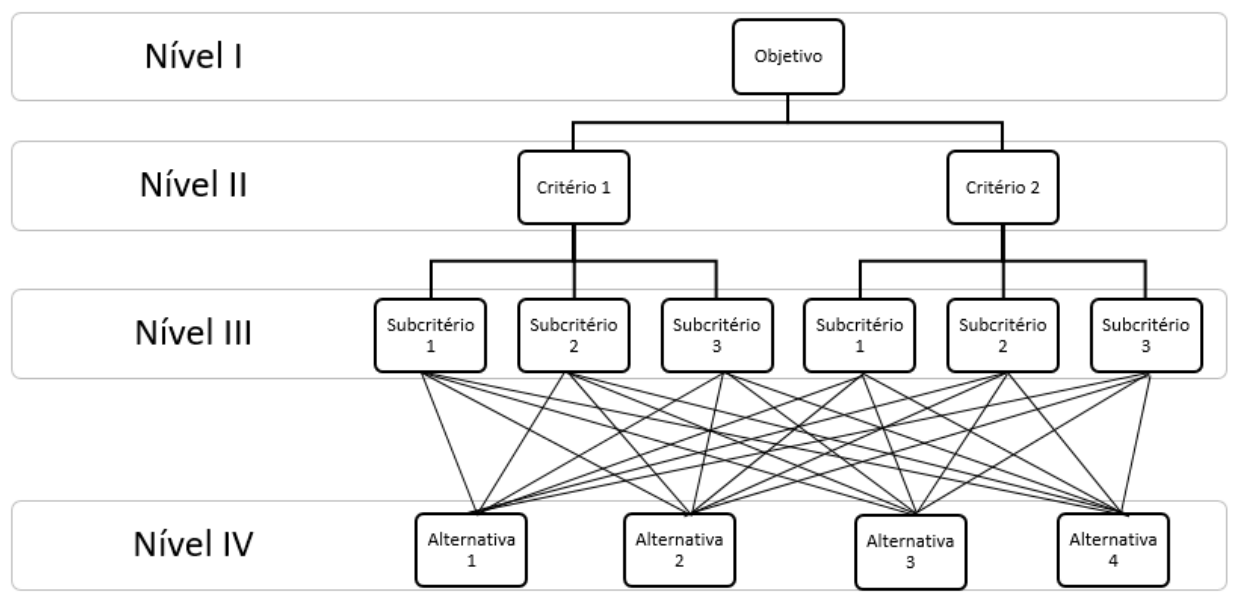

Fuente: adaptado de [22].

En la segunda etapa, los juicios comparativos, los especialistas evalúan la importancia de los atributos. Esto se logra asociando ponderaciones para cada elemento a través de comparaciones emparejadas. La tercera etapa consiste en la síntesis de prioridades, es decir, la estimación final del peso de cada alternativa.

En términos matemáticos, la función de valor para cada alternativa es dada por la Ecuación (1):

$$
F(a)=\sum_{j=1}^{n} w_{j} v_{j}(a)
$$

En esta ecuación:

$F(a)$ : indica el valor final de la alternativa a;

$w_{j}$ : denota el peso del j-ésimo criterio;

$v_{j}$ : denota el desempeño de la alternativa en relación al j-ésimo criterio.

Una vez realizadas las comparaciones, las respuestas se organizan en una matriz donde las filas y columnas representan los elementos en comparación, en este sentido, la matriz montada siempre será cuadrada y recíproca (Ecuación 2):

$$
A w=\lambda_{\max } w
$$

En esta ecuación:

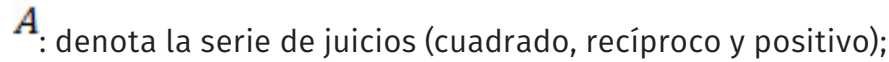

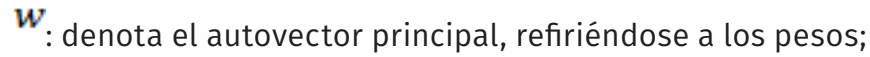

$\lambda_{\max }:$ denota el valor principal de A

El siguiente es un desarrollo simple e intuitivo para entender el análisis de coherencia [25]. Suponiendo una matriz coherente, donde las comparaciones se basan en mediciones exactas, es decir: los pesos $w_{1}, w_{2}, \ldots, w_{n}$ ya se conocen. Por lo tanto, la Ecuación (3) presenta esta situación: 


$$
a_{i j}=\frac{w_{i}}{w_{j}}
$$

Como el ensayo es perfecto para todas las comparaciones, tiene que $a_{i k}=a_{i j} \cdot a_{j k}$ para cualquier i, j, $\mathrm{k}$, que va de 1 a n, siendo $\mathrm{n}$ el orden de la matriz. En este sentido, la siguiente expresión también es válida:

$$
a_{i j}=\frac{w_{j}}{w_{i}}=\frac{1}{w_{i} / w_{j}}
$$

Considerando $x=\left(x_{1}, x_{2}, \ldots x_{n}\right)$ e $y=\left(y_{1}, y_{2}, \ldots, y_{n}\right)$ se puede escribir en una notación de matriz $A \cdot x=y$, donde A es la matriz de juicios, dada por:

$$
A=\left[\begin{array}{cccc}
\frac{w_{1}}{w_{1}} & \frac{w_{1}}{w_{2}} & \ldots & \frac{w_{1}}{w_{n}} \\
w_{2} & \frac{w_{2}}{w_{2}} & \ldots & \frac{w_{2}}{w_{n}} \\
\vdots & w_{2} & \ddots & \vdots \\
\vdots & w_{n} & \ldots & \frac{w_{n}}{w_{n}}
\end{array}\right]
$$

Algebraicamente esta operación se puede representar mediante la Ecuación (6):

$$
\sum_{j=0}^{n} a_{i j} \cdot x_{i}=y_{i} \quad \therefore \quad i=1,2,3, \ldots, n
$$

Dado que $a_{i j}=\frac{w_{j}}{w_{i}}$, se obtiene:

$$
a_{i j} \frac{w_{j}}{w_{i}}=1 \quad \therefore \quad i, j=1,2,3, \ldots, n
$$

Por consiguiente:

$$
\sum_{j=0}^{n} a_{i j} \cdot w_{j} \frac{1}{w_{i}}=n \quad \therefore \quad i=1,2,3, \ldots, n
$$

O:

$$
\sum_{j=0}^{n} a_{i j} \cdot w_{j}=n w_{i} \quad \therefore \quad i=1,2,3, \ldots, n
$$

Que es equivalente a la ecuación de matriz:

$$
A w=n w
$$


La Ecuación (10), en álgebra lineal, indica que w es autovector de A con autovalor n. En la práctica $a_{i j}$ son los pesos atribuidos por el juicio de los expertos, basado según la escala fundamental, y de cierta manera subjetiva. Por lo tanto, los valores de $a_{i j}$ se alejarán del "ideal" $w_{i} / w_{j}$, haciendo que la ecuación $A w=n w$ ya no sea válida.

Si el valor de $\lambda_{1}, \lambda_{2}, \ldots, \lambda_{n}$ son los números que satisfacen la ecuación $A w=\lambda w$, entonces $\lambda$ es autovalor de $\mathrm{A}$ y, si $a_{i j}=1$ para todo i, entonces:

$$
\sum_{i=0}^{n} \lambda_{i}=n
$$

El valor de interés para el desarrollo de la metodología es el autovector normalizado, por lo que la suma de w es igual a 1. Para esto cada elemento wi se divide por su suma.

Una medida de consistencia, llamada índice de consistencia (IC) dada por la ecuación (12), se utiliza para calcular la desviación de $\lambda_{\max }$ en relación con n, ya que el uso de la escala para las sentencias genera variaciones en $a_{i j}$, alterando el valor de $\lambda_{\max }$ :

$$
I C=\frac{\lambda_{\max }-n}{n-1}
$$

En esta metodología AHP, es común que las evaluaciones realizadas por los especialistas generen incoherencias, esto es parte del juicio humano [26]. Sin embargo, se desea que sean los más pequeños posibles [27]. La relación de consistencia se utiliza, como se mencionó anteriormente, dada por la siguiente expresión:

$$
R C=\frac{I C}{I R}
$$

El parámetro IR, también conocido como Índice aleatorio, denota el índice de consistencia de una matriz recíproca generada aleatoriamente, basada en la escala de 1 a 9, como recíprocos forzados. Este valor es tabulado y varía según el orden de la matriz [28].

La evaluación final de la coherencia de la sentencia se produce al comparar el valor de RC. Para el desarrollo de la investigación, se hacen las siguientes consideraciones: $R C \leq 0,1$ : consiste en un juicio coherente; $0,1<R C<0,2$ : juicio cuestionable; $R C \geq 0,2$ : sentencia incoherente.

Una vez determinados los pesos de los criterios y alternativas de las estrategias de las tecnologías para generación de energía eléctrica a partir de fuentes renovables, la fase II consiste en la aplicación del método TOPSIS (Technique for Order of Preference by Similarity to Ideal Solution) que fue introducido por Yoon y Hwang, en 1981 [29]. A continuación, se describe la formulación matemática del método TOPSIS, que se basa en la aplicación de 7 etapas o fases.

Sea la matriz de decisión D compuesta de alternativa "m" evaluada por criterios "n":

$$
D=\left[\begin{array}{cccc}
X_{11} & X_{12} & \ldots & X_{1 n} \\
X_{21} & X_{22} & \ldots & \\
\vdots & \vdots & \ddots & X_{2 n} \\
X_{m 1} & X_{m 2} & \ldots & X_{m n}
\end{array}\right]
$$


El problema abordado debe alcanzar las siguientes, fundamentalmente, tres condiciones: (i) A1, A2, ..., Am son alternativas viables; (ii) C1, C2, ..., Cn son criterios; (iii) Xij indica el rendimiento de la alternativa Aij de acuerdo con el criterio $\mathrm{Cj}$.

- Fase 1: Los datos de la Matrix A tiene orígenes diferentes, por lo que debe normalizarse para convertirlos en una matriz dimensional para que sea posible comparar varios criterios. Para normalizarlo, se utiliza la siguiente ecuación:

$$
p_{i j}=\frac{x_{i j}}{\max _{x i}}
$$

En la ecuación de arriba, $\mathrm{j}=1, \ldots . \mathrm{n}$ representa el valor máximo de Xi para cada criterio Cj. La matriz Dn representa la matriz de decisiones normalizada.

$$
D_{n}=\left[\begin{array}{cccc}
p_{11} & p_{12} & \cdots & p_{1 n} \\
p_{21} & p_{22} & \cdots & \\
\vdots & \vdots & \ddots & p_{2 n} \\
p_{m 1} & p_{m 2} & \cdots & p_{m n}
\end{array}\right]
$$

- Fase 2: en esta etapa el objetivo es calcular los pesos de cada uno de los criterios adoptados. Por lo tanto, sea el vector de peso $\mathrm{W}$ compuesto por los pesos individuales $\mathrm{Wj}(\mathrm{j}=1, \ldots, \mathrm{n})$ para cada criterio Cj cumple. Por lo tanto, cada criterio j tendrá un peso, obteniendo así el vector de los pesos dados por la siguiente expresión:

$$
W=\left[W_{1}, W_{2}, \ldots, W_{n}\right]
$$

En el modelo TOPSIS clásico, los pesos se calculan por entropía representando la cantidad de información de la matriz de decisión:

$$
e_{j}=-\frac{1}{\ln (m)} \sum_{i=1}^{m} p_{i j} \ln \left(p_{i j}\right)
$$

Siendo "m" el número de alternativas, i = 1...n, j = 1...m. El grado de diversidad de la información contenida en cada criterio se calcula utilizando la siguiente ecuación:

$$
d_{j}=1-e_{j}
$$

El peso de cada criterio por el método de entropía se calcula de la siguiente manera:

$$
W_{j}=d_{j} / \sum_{j=1}^{n} d_{j}
$$

- Fase 3: en esta etapa deben calcularse los valores normalizados por los pesos, de acuerdo con la siguiente ecuación:

$$
V_{i j}=W_{i j} \cdot p_{i j}
$$


Por lo tanto, la matriz de decisión se normaliza por los pesos de cada criterio $D_{n p}$

$$
D_{n}=\left[\begin{array}{cccc}
V_{11} & V_{12} & \cdots & V_{1 n} \\
V_{21} & V_{22} & \cdots & \\
\vdots & \vdots & \ddots & V_{2 n} \\
V_{m 1} & V_{m 2} & \cdots & V_{m n}
\end{array}\right]
$$

La matriz anterior representa el rendimiento relativo de las alternativas.

- Fase 4: en esta etapa, el cálculo de las soluciones positivas ideales A+ (beneficios) y las soluciones ideales negativas A- (costos) se calculan de acuerdo con las siguientes expresiones:

$$
\begin{aligned}
& A^{+}=\left[V_{1}^{+}, V_{2}^{+}, \ldots, V_{n}^{+}\right]=\left[\left(\max _{j} V_{i j} \mid j=1, \ldots, n\right) \mid i=1, \ldots, m\right] \\
& A^{-}=\left[V_{1}^{-}, V_{2}^{-}, \ldots, V_{n}^{-}\right]=\left[\left(\min _{j} V_{i j} \mid j=1, \ldots, n\right) \mid i=1, \ldots, m\right]
\end{aligned}
$$

- Fase 5: A continuación, las distancias euclidianas entre Ai y A+ y entre Ai y A- deben calcularse mediante las siguientes expresiones:

$$
\begin{gathered}
d^{+}=\sqrt{\sum_{j=1}^{n} W_{j}\left(V_{i j}-V_{j}^{+}\right)^{2}} \\
d^{-}=\sqrt{\sum_{j=1}^{n} W_{j}\left(V_{i j}-V_{j}^{-}\right)^{2}}
\end{gathered}
$$

- Fase 6: En esta etapa se debe calcular la similitud relativa para cada Alternativa Ai en relación con la solución ideal positiva y la solución ideal negativa, según la ecuación:

$$
\xi_{i}=\frac{d_{i}^{-}}{d_{i}^{-}+d_{i}^{+}}
$$

- Fase 7: finalmente, en esta última fase, las alternativas se clasifican según la similitud relativa (clasificación). Las mejores alternativas son aquellas que tienen los valores de similitudes relativas más altos. En este sentido, deben elegirse estas alternativas, ya que están más cerca de la solución positiva ideal. 


\section{Resultados}

A partir de la información disponible en la literatura especializada sobre publicaciones de distintos autores en el área de investigación [30,31,32], fue percibido que el estudio de fuentes renovables para la generación de energía eléctrica implica criterios ambientales, sociales, políticos, técnicos, riesgos, sociopolíticos, entre otros. La Tabla 1 consolida los artículos analizados y los criterios que los diversos autores proponen en su investigación.

Tabla 1 - Criterios adoptados en investigaciones publicadas y disponibles en la literatura

\begin{tabular}{|c|c|}
\hline Autores & Criterios \\
\hline Streimikiene et al. [33]. & Ambiental, económico, ético, político y técnico. \\
\hline Al Garni et al. [34]. & Ambiental, económico, sociopolítico y técnico. \\
\hline Shmelev \& Van Den Bergh [35]. & $\begin{array}{c}\text { Ambiental, económico, entradas de recursos, } \\
\text { riesgos, social y técnico. }\end{array}$ \\
\hline Hadian \& Madani [36]. & Ambiental, económico e social. \\
\hline Arcer et al. [37]. & Ambiental, económico y técnico. \\
\hline Şengül et al. [38]. & Ambiental, económico y técnico. \\
\hline Troldborg et al. [39]. & Ambiental, socioeconómico y técnico. \\
\hline Santoyo-Castelazo \& Azapagic [40]. & Ambiental, económico y técnico. \\
\hline Mourmouris \& Potolias [41]. & Ambiental, económico y técnico. \\
\hline Baris \& Kucucali [42]. & Ambiental, económico y técnico. \\
\hline Kaya \& Kahraman [43]. & Ambiental, económico, social y técnico. \\
\hline
\end{tabular}

Fuente: elaboración propia

A partir de la información recolectada y consolidada en la Tabla 1, fueron seleccionados tres criterios los cuales son citados de forma amplia en la literatura especializada, estos son: (i) Económico, (ii) Ambiental y (iii) Técnico.

Con relación al proceso de recolección y evaluación de datos, el instrumento de investigación consideró nueve temas sobre energía renovable, teniendo en cuenta las características inherentes de las diferentes fuentes para la generación de energía de eléctrica, así como los criterios y subcriterios de análisis adoptados. En general, los temas se agruparon en tres dimensiones: económico, ambiental y técnico. La Tabla 2 resume los temas y dimensiones considerados en la investigación.

Tabla 2 - Criterios adoptados en para el desarrollo de la investigación

\begin{tabular}{|c|c|c|}
\hline \multirow{2}{*}{ Critério } & \multicolumn{2}{c|}{ Subcritérios } \\
\hline \multirow{4}{*}{ Económico } & E1 & Costo del nivel de energía \\
\cline { 2 - 3 } & E2 & Retorno de la inversión (Payback) \\
\cline { 2 - 3 } & E3 & Riesgo de inversión \\
\hline \multirow{4}{*}{ Ambiental } & A1 & Emisiones de gases de efecto invernadero \\
\cline { 2 - 3 } & A2 & Consumo de agua \\
\cline { 2 - 3 } & A3 & Generación de residuos al final del ciclo de vida \\
\hline \multirow{3}{*}{ Técnico } & T1 & Rendimiento tecnológico \\
\cline { 2 - 3 } & T2 & Disponibilidad de recursos potenciales \\
\cline { 2 - 3 } & T3 & Distribución descentralizada \\
\hline
\end{tabular}

Fuente: elaboración propia 
Para la evaluación de las alternativas, se adoptó una escala Likert de 5 puntos para cada pregunta, con el fin de que los especialistas evaluaran el nivel de madurez en relación con cada uno de los 9 temas. Los niveles de madurez considerados fueron: 1-Muy bajo; 2-bajo; 3-Moderado; 4-alto; 5-Muy alto.

A continuación, las Tablas 3 y 4 presentan los resultados de las matrices obtenidas:

Tabla 3 - Matriz de comparación criterios

\begin{tabular}{|c|c|c|c|c|c|c|c|c|c|c|c|c|c|c|c|c|c|c|c|c|c|}
\hline \multirow{2}{*}{ Criterios } & \multicolumn{7}{|c|}{ E } & \multicolumn{7}{|c|}{ A } & \multicolumn{7}{|c|}{$T$} \\
\hline & & l & & m & & u & & & I & & m & & t & & & I & & m & & u & \\
\hline $\mathrm{E}$ & ( & 1 & ; & 1 & ; & 1 & ) & ( & 4 & ; & 5 & ; & 6 & ) & ( & & ; & 3 & & 4 & ) \\
\hline A & ( & $1 / 6$ & ; & $1 / 5$ & ; & $1 / 4$ & ) & ( & 1 & ; & 1 & ; & 1 & ) & 1 & $1 / 5$ & ; & $1 / 4$ & ; & $1 / 3$ & ) \\
\hline $\mathrm{T}$ & ( & $1 / 4$ & ; & $1 / 3$ & ; & $1 / 2$ & ) & ( & 3 & ; & 4 & ; & 5 & ) & ( & 1 & ; & 1 & ; & 1 & ) \\
\hline
\end{tabular}

E: Económico; A: Ambiental; T: Técnico ; l: límite inferior; m: mediana; $\mathbf{u}$ : límite superior

\section{Fuente: elaboración propia}

Tabla 4 - Matriz de comparación: subcriterios Económico, Ambiental y Técnico

\begin{tabular}{|c|c|c|c|c|c|c|c|c|c|c|c|c|c|c|c|c|c|c|c|c|c|c|}
\hline \multirow{2}{*}{ Criterio } & \multirow{2}{*}{ Subcriterios } & \multicolumn{7}{|c|}{ E1 } & \multicolumn{7}{|c|}{ E2 } & \multicolumn{7}{|c|}{ E3 } \\
\hline & & & l & & $\mathbf{m}$ & & $\mathbf{u}$ & & & I & & m & & $\mathbf{u}$ & & & I & & m & & $\mathbf{u}$ & \\
\hline \multirow{3}{*}{ Económico } & E1 & ( & 1 & ; & 1 & ; & 1 & ) & ( & $1 / 5$ & ; & $1 / 4$ & ; & $1 / 3$ & ) & ( & $1 / 8$ & ; & $1 / 7$ & ; & $1 / 6$ & ) \\
\hline & E2 & ( & 3 & ; & 4 & ; & 5 & ) & ( & 1 & ; & 1 & ; & 1 & ) & ( & $1 / 4$ & ; & $1 / 3$ & ; & $1 / 2$ & ) \\
\hline & E3 & ( & 6 & ; & 7 & ; & 8 & ) & ( & 2 & ; & 3 & ; & 4 & ) & ( & 1 & ; & 1 & ; & 1 & ) \\
\hline \multirow{3}{*}{ Ambiental } & $\mathrm{A} 1$ & ( & 1 & ; & 1 & ; & 1 & ) & ( & $1 / 9$ & ; & $1 / 8$ & ; & $1 / 7$ & ) & ( & $1 / 6$ & ; & $1 / 5$ & ; & $1 / 4$ & ) \\
\hline & A2 & ( & 7 & ; & 8 & ; & 9 & ) & ( & 1 & ; & 1 & ; & 1 & ) & ( & 2 & ; & 3 & ; & 4 & ) \\
\hline & A3 & ( & 4 & ; & 5 & ; & 6 & ) & ( & $1 / 4$ & ; & $1 / 3$ & ; & $1 / 2$ & ) & ( & 1 & ; & 1 & ; & 1 & ) \\
\hline \multirow{3}{*}{ Técnico } & $\mathrm{T} 1$ & ( & 1 & ; & 1 & ; & 1 & ) & ( & 8 & ; & 9 & ; & 9 & ) & ( & 1 & ; & 2 & ; & 3 & ) \\
\hline & $\mathrm{T} 2$ & ( & $1 / 9$ & ; & $1 / 9$ & ; & $1 / 8$ & ) & ( & 1 & ; & 1 & ; & 1 & ) & ( & $1 / 4$ & ; & $1 / 3$ & ; & $1 / 2$ & ) \\
\hline & T3 & ( & $1 / 3$ & ; & $1 / 2$ & ; & 1 & ) & ( & 2 & ; & 3 & ; & 4 & ) & ( & 1 & ; & 1 & ; & 1 & ) \\
\hline
\end{tabular}

Fuente: elaboración propia

Se desarrolló un algoritmo en Microsoft Excel y, a partir de la evaluación de matrices crips, fue posible realizar un análisis de consistencia de matrices de comparación pareadas. Los resultados mostraron que Îndice de Consistencia (RC), para las matrices de criterios y subcriterios, atienden la condición de $\mathrm{RC} \leq 0,10$. Una vez que se confirmó la consistencia de todas las matrices, se obtuvieron los pesos de los criterios y subcriterios mediante el método AHP. Los pesos se determinaron mediante el método de la media geométrica, obteniéndose los siguientes resultados: Criterio Económico (0.8221); Criterio Ambiental (0.1228); Criterio Técnico (0.3669).

En ese sentido, los resultados mostraron que el criterio más relevante al momento de implementar una alternativa para la generación de energía eléctrica a partir de fuentes renovables está asociado al aspecto económico. Los especialistas afirman que la economía global está sufriendo un dinamismo acelerado, así como una inestabilidad poco usual en los últimos 20 años. Sin duda, Colombia es afectada por estas situaciones. Además, aspectos sociales y corrientes políticas influencian directamente en la Economía del País. Por esta razón, los especialistas coinciden en afirmar de forma cualitativa, además de ser verificado cuantitativamente, que un cuidado especial debe ser considerado a las directrices económicas con el 
propósito de implementar estrategias eficaces para la generación de energía eléctrica a partir de fuentes renovables. Una vez conocido el peso de cada criterio, la Tabla 5 evalúa los resultados para cada subcriterio.

Tabla 5 - Pesos de los subcriterios por el método AHP

\begin{tabular}{|c|c|c|c|c|c|c|c|c|c|}
\hline \multirow{2}{*}{ Critério } & \multirow{2}{*}{\multicolumn{2}{|c|}{ Subcritérios }} & \multicolumn{7}{|c|}{ Peso } \\
\hline & & & & $\mathbf{L}$ & & M & & $\mathbf{U}$ & \\
\hline \multirow{3}{*}{ Económico } & E1 & Costo del nivel de energía & ( & 0.0975 & ; & 0.1098 & ; & 0.1272 & ) \\
\hline & E2 & Retorno de la inversión (Payback) & ( & 0.3029 & ; & 0.3669 & ; & 0.4524 & ) \\
\hline & E3 & Riesgo de inversión & ( & 0.7631 & ; & 0.9196 & ; & 1.0583 & ) \\
\hline \multirow{3}{*}{ Ambiental } & $\mathrm{A} 1$ & Emisiones de gases de efecto invernadero & ( & 0.0882 & ; & 0.0975 & ; & 0.1098 & ) \\
\hline & $\mathrm{A} 2$ & Consumo de agua & ( & 0.8034 & ; & 0.9615 & ; & 1.1006 & ) \\
\hline & A3 & $\begin{array}{l}\text { Generación de residuos al final del ciclo de } \\
\text { vida }\end{array}$ & ( & 0.3333 & ; & 0.3952 & ; & 0.4807 & ) \\
\hline \multirow{3}{*}{ Técnico } & $\mathrm{T} 1$ & Rendimiento tecnológico & ( & 0.6667 & ; & 0.8736 & ; & 1.0000 & ) \\
\hline & $\mathrm{T} 2$ & Disponibilidad de recursos potenciales & ( & 0.1010 & ; & 0.1111 & ; & 0.1323 & ) \\
\hline & $\mathrm{T} 3$ & Distribución descentralizada & ( & 0.2912 & ; & 0.3816 & ; & 0.5291 & ) \\
\hline
\end{tabular}

Fuente: elaboración propia

La Tabla 5 mostró, de forma evidente, que existen tres subcriterios críticos que deben ser considerados en cada uno de los criterios analizados, en su orden: Riesgo de inversión (Económico), Rendimiento tecnológico (Técnico) y Consumo de agua (Ambiental). Los especialistas coinciden en afirmar que el riesgo de la inversión inicial, en un mercado fluctuante, es un aspecto fundamental que incide fuertemente a la hora de tomar una decisión de implementar fuentes alternativas para la generación de energía eléctrica. Adicionalmente, el desempeño técnico que tendrá la opción elegida, en relación a su eficiencia energética es el principal aspecto a tener en cuenta en el criterio técnico. Es decir, la capacidad que tendrá la alternativa tecnológica en transformar su fuente primaria de energía en energía eléctrica y conseguir atender la demanda en la costa caribe, es una inquietud que se deben analizar con detalle. Finalmente, desde el punto de vista ambiental, los especialistas manifiestan una especial preocupación por el consumo del agua que pueda producir una fuente alternativa para la generación de energía eléctrica. Este recurso natural es el principal aspecto a "cuidar" al momento de implementar una alternativa para la generación de energía eléctrica. De esta forma se promueve la preservación del medio ambiente y, al mismo tiempo, se está alineando al País con las políticas de sostenibilidad internacional.

Con los criterios y los pesos de los subcriterios definidos, a continuación, se comienza la Fase TOPSIS. A partir de los resultados de los pesos de los criterios y subcriterios, se construyó la matriz de decisión $\widetilde{D}$. Luego, la multiplicación de los pesos de los criterios por el peso de los subcriterios, resulta el vector peso total $W_{\text {Total }}$, conforme se muestra en la Tabla 6. 
Tabla 6 - Peso total de los subcriterios por el método AHP $\left(\boldsymbol{W}_{T \text { otal }}\right)$

\begin{tabular}{|c|c|c|c|c|c|c|c|c|c|}
\hline \multirow{2}{*}{ Critério } & \multirow{2}{*}{\multicolumn{2}{|c|}{ Subcritérios }} & \multicolumn{7}{|c|}{ Peso Total } \\
\hline & & & & L & & M & & U & \\
\hline \multirow{3}{*}{ Económico } & E1 & Costo del nivel de energía & ( & 0.0650 & ; & 0.0902 & ; & 0.1223 & ) \\
\hline & E2 & Retorno de la inversión (Payback) & ( & 0.2019 & ; & 0.3016 & ; & 0.4350 & ) \\
\hline & E3 & Riesgo de inversión & ( & 0.5088 & ; & 0.7560 & ; & 1.0175 & ) \\
\hline \multirow{3}{*}{ Ambiental } & A1 & Emisiones de gases de efecto invernadero & ( & 0.0095 & ; & 0.0120 & ; & 0.0160 & ) \\
\hline & A2 & Consumo de agua & ( & 0.0862 & ; & 0.1181 & ; & 0.1602 & ) \\
\hline & A3 & Generación de residuos al final del ciclo de vida & ( & 0.0358 & ; & 0.0485 & ; & 0.0700 & ) \\
\hline \multirow{3}{*}{ Técnico } & $\mathrm{T} 1$ & Rendimiento tecnológico & ( & 0.2019 & ; & 0.3205 & ; & 0.4524 & ) \\
\hline & $\mathrm{T} 2$ & Disponibilidad de recursos potenciales & ( & 0.0306 & ; & 0.0408 & ; & 0.0598 & ) \\
\hline & T3 & Distribución descentralizada & ( & 0.0882 & ; & 0.1400 & ; & 0.2394 & ) \\
\hline
\end{tabular}

Fuente: elaboración propia

Los resultados de la Tabla 6 son coherentes con los resultados de los pesos de los criterios mostrados en la Tabla 5. Esta Tabla 9 mostró el peso de los subcriterios ponderado por el peso de su respectivo criterio. Esto confirma, una vez más, que los subcriterios de mayor importancia que deben ser analizados, en su orden, son: Riesgo de la inversión, Rendimiento Tecnológico y Consumo del agua.

Continuando con la aplicación del método, la matriz de evaluación de decisión $\widetilde{\boldsymbol{D}}$ es normalizada y la matriz normalizada ponderada $\widetilde{\boldsymbol{V}}$ es calculada. A partir de los resultados y la matriz normalizada y ponderada por el método AHP es posible determinar la solución ideal positiva y negativa (PIS e NIS), considerando los valores máximos y mínimos de cada subcriterio. Luego, se estimó la proximidad relativa a la solución ideal. Este valor fue calculado a partir de las distancias totales positivas y negativas, cuya representación es dada por el coeficiente de proximidad (CCi). Este parámetro representa el índice de desempeño de cada alternativa a partir de la evaluación de los tres criterios: económico, ambiental y técnico. La Tabla 7 consolida los resultados para el cálculo del coeficiente de proximidad.

Tabla 7 - Cálculo del coeficiente de proximidad

\begin{tabular}{|c|c|c|c|c|}
\hline \multicolumn{2}{|c|}{ Alternativas } & Distancias & \multirow{2}{*}{ CCi } \\
\cline { 2 - 4 } & D+ & D- & \\
\hline Alternativa 1 & Hidráulica & 2.6310 & 2.2201 & 0.4576 \\
\hline Alternativa 2 & Fotovoltaica & 2.4036 & 2.4099 & 0.5006 \\
\hline Alternativa 3 & Eólica & 2.4605 & 2.3338 & 0.4868 \\
\hline Alternativa 4 & Biomasa & 2.4498 & 2.4355 & 0.4985 \\
\hline Alternativa 5 & Onda & 2.4899 & 2.3434 & 0.4848 \\
\hline
\end{tabular}

\section{Fuente: elaboración propia}

Conforme el resultado mostrado en la tabla anterior, en función del coeficiente de proximidad (CCi), el orden de las alternativas propuestas, de mayor a menor corresponde a: Fotovoltaica (Alternativa 2), Biomasa (Alternativa 4), Eólica (Alternativa 3), Onda (Alternativa 5), Hidráulica (Alternativa 1). Para una mejor visualización de los resultados, a seguir la Figura 2 ilustra el gráfico radar que resalta el CCi para cada alternativa propuesta. 
Figura 2 - Gráfico radar con la identificación del CCi para cada alternativa

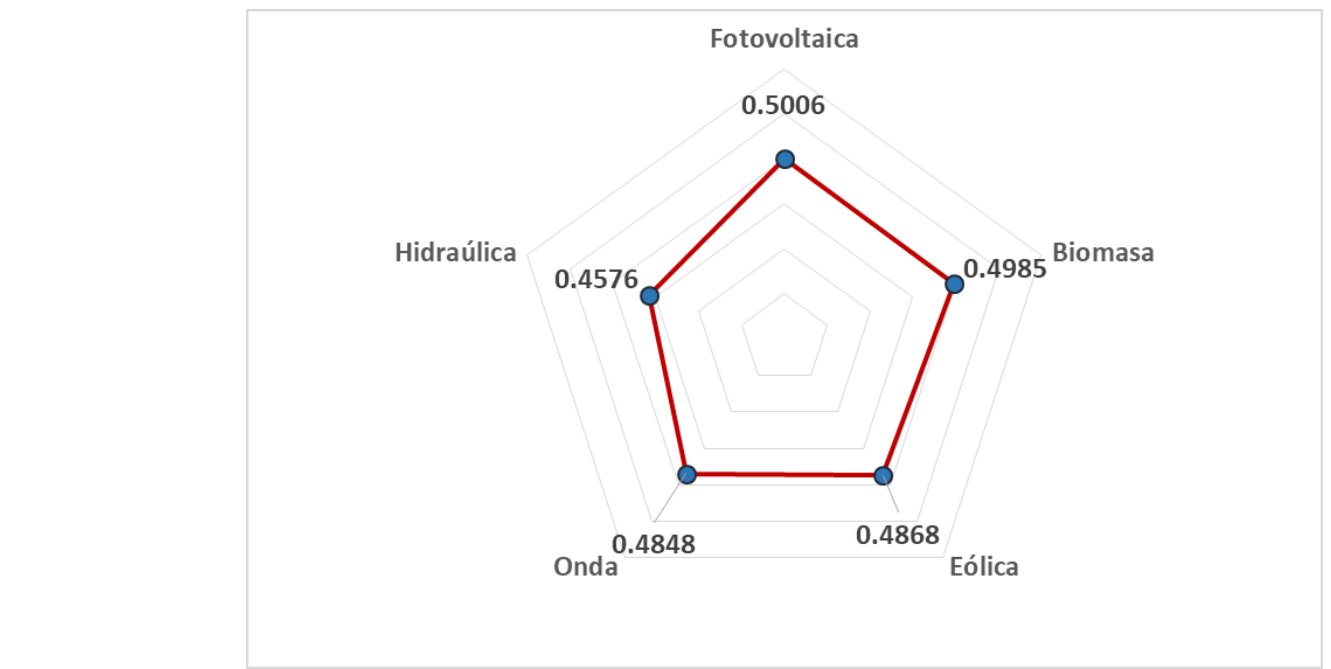

Fuente: elaboración propia

En general, los resultados de la Figura 2 muestran que los índices de las alternativas 2 (Fotovoltaica), 3 (Eólica) y 4 (Biomasa), se encuentra por el encima de la media aritmética $(0,4857)$. Por otro lado, la alternativa 2 , se encuentra por debajo del segundo cuartil $(0,4868)$. Finalmente, la alternativa 1 , se encuentra por debajo del primer cuartil $(0,4712)$.

\section{Conclusiones}

Este trabajo permitió evaluar las mejores alternativas para la generación de energía eléctrica a partir de fuentes renovables. Fue posible confirmar que el criterio económico es aquel que mayor impacto tiene al momento de seleccionar una alternativa para la generación de energía. Adicionalmente, el Riesgo de la Inversión, fue rankeado como el subcriterio más crítico al momento de seleccionar una determinada alternativa de generación de energía eléctrica. Adicionalmente, fue confirmado que el dinamismo del mercado internacional, diversas políticas sustentables, así como la actual crisis política que repercute en los países latinoamericanos permiten explicar la inestabilidad de la economía en pro de invertir en alternativas tecnológicas. Razón por la cual, el criterio económico y el subcriterio relacionado al riesgo de la inversión fueron considerados los más críticos en el estudio realizado y, consecuentemente, son las principales barreras a ser superadas con el propósito de viabilizar la implementación de fuentes de energías limpias en la matriz energética colombiana. 


\section{Referencias Bibliográficas}

1. C. Díaz-Rodríguez, "Aspectos bioéticos relacionados con la producción y demanda residencial de energía eléctrica en Colombia," 2015.

2. X. Xu, Z. Wei, Q. Ji, C. Wang, and G. Gao, "Global renewable energy development: Influencing factors, trend predictions and countermeasures," Resources Policy, vol. 63, p. 101470, 2019.

3. E. D. Kocak, M. Tasdemir, and C. Gul, "Sustainable Green-Based Composites from Renewable Resources in Textile: Industrial Cotton Wastes," in Sustainability in the Textile and Apparel Industries: Springer, 2020, pp. 45-61.

4. Y. Wang, L. Xu, and Y. A. Solangi, "Strategic renewable energy resources selection for Pakistan: Based on SWOT-Fuzzy AHP approach," Sustainable Cities and Society, vol. 52, p. 101861, 2020.

5. D. Thrän, K. Schaubach, S. Majer, and T. Horschig, "Governance of sustainability in the German biogas sector-Adaptive management of the Renewable Energy Act between agriculture and the energy sector," Energy, Sustainability and Society, vol. 10, no. 1, p. 3, 2020.

6. K. Hashii, Y. Yamahata, and T. Suzuki, "Evaluation and care protocol for maternal emergencies: the basic J-CIMELS protocol derived from the Kyoto protocol," Hypertension Research in Pregnancy, vol. 8, no. 1, pp. 4-14, 2020.

7. A. Mikhaylov, N. Moiseev, K. Aleshin, and T. Burkhardt, "Global climate change and greenhouse effect," Entrepreneurship and Sustainability Issues, vol. 7, no. 4, pp. 2897-2913, 2020.

8. M. E. Madrid Wolff and J. M. Toro Bedoya, "Viabilidad técnica y económica de tornillos hidrodinámicos para generación eléctrica. Caso Quebrada El Chuscal," Universidad EIA, 2013.

9. F. I. Cuervo, "Valoración de fuentes renovables no convencionales de generación de electricidad: un enfoque desde las opciones reales," Cuadernos de Administración, vol. 28, no. 51, pp. 45-64, 2015.

10. L. Beltrán, "Análisis de los diferentes tipos de energías alternativas y su implementación en Colombia," thesis, Universidad Militar Nueva Granada, Bogotá, 2016.

11. M. Castro, P. Castro, and M. Cunha, "Análisis Comparativo de Indicadores Energéticos de Países Miembros de la Comunidad Andina de Naciones," Revista Técnica" energía", vol. 14, no. 1, pp. 236-245 pp., 2018.

12. G. Corredor, "Colombia y la transición energética," Ciencia política, vol. 13, no. 25, pp. 107-125, 2018.

13. C. Montes, "La incertidumbre climática y el dilema energético colombiano," Revista de la Academia Colombiana de Ciencias Exactas, Físicas y Naturales, vol. 42, no. 165, pp. 392-401, 2018.

14. G. Romero et al., "Implicaciones de política del Acuerdo de París en la planeación del sistema eléctrico de Colombia," Departamento Nacional de Planeación, 2018.

15. R. Moreno, Y. López, and E. C. Q. Oqueña, "Escenario de Desarrollo Energético Sostenible en Colombia 2017-2030," Avances: Investigacion en Ingeniería, vol. 15, no. 1, pp. 329-343, 2018.

16. C. D. Ocampo, J. Tamayo, and H. M. Castaño, "Gestión del Riesgo en la Implementación de Sistemas Fotovoltaicos en Proyectos de Extracción de Oro en Colombia a partir del Proceso de Análisis Jerárquico (AHP)," Información tecnológica, vol. 30, no. 3, pp. 127-136, 2019.

17. G. Romero, A. Álvarez-Espinosa, S. Calderón, and A. Ordóñez, "Redistributive impacts of a carbon tax in Colombia: the link between models of microsimulations and general equilibrium," Lecturas de Economía, no. 89, pp. 163-198, 2018. 
18. H. Porras, A. Martínez, and M. Herrera, "Un análisis de las implicaciones de la falta de cobertura de energía renovable no convencional en Colombia," Revista Inventum, vol. 13, no. 25, p. 41, 2018.

19. F. J. Rojas, S. Pacsi, O. R. Sánchez-Ccoyllo, and M. M. Perales, "Pronóstico de Reducción de Emisiones, de Enfermos y de Gastos Asociados al incluir el Gas Natural dentro de la Matriz Energética en Perú," Información tecnológica, vol. 30, no. 3, pp. 117-126, 2019.

20. S. M. Rincón and A. K. V. Camargo, "Energías renovables un futuro optimo para Colombia," Punto de vista, vol. 9, no. 13, p. 2, 2018.

21. J. Solano, F. Ferreira. Evaluación del desempeño de tecnologías para la generación de energía eléctrica a partir de fuentes renovables en la costa caribe colombiana utilizando el modelo multicriterio FuzzyAHP-TOPSIS. Puerto Colombia, 2019. 85p. Trabajo de grado. Programa de Ingeniería Electromecánica (FIMEB). Universidad Antonio Nariño

22. T. L. Saaty, "A scaling method for priorities in hierarchical structures," Journal of mathematical psychology, vol. 15, no. 3, pp. 234-281, 1977.

23. L. THOMAS and L. Vargas, "Decision Making with the Analytic Network Process," ed: German: Springer, 2006.

24. N. Kadoi, N. Reep, B. Divjak. Toma de decisiones con el proceso de la red analítica. $14^{\circ}$ Simposio Internacional de Investigación Operativa en Eslovenia, 2017.

25. T. L. Saaty, "Decision making for leaders," IEEE Transactions on Systems, Man, and Cybernetics, no. 3, pp. 450-452, 1985.

26. M. A. O. Barrios, F. De Felice, K. P. Negrete, B. A. Romero, A. Y. Arenas, and A. Petrillo, "An AHP-Topsis Integrated Model for Selecting the Most Appropriate Tomography Equipment," International Journal of Information Technology \& Decision Making, vol. 15, no. 04, pp. 861-885, 2016.

27. H. G. Costa, "Auxílio multicritério à decisão: método AHP," Rio de Janeiro: Abepro, 2006.

28. R. Csutora and J. J. Buckley, "Fuzzy hierarchical analysis: the Lambda-Max method," Fuzzy sets and Systems, vol. 120, no. 2, pp. 181-195, 2001.

29. K. Yoon and C.-L. Hwang, Multiple attribute decision making: methods and applications. SPRINGERVERLAG BERLIN AN, 1981.

30. J. E. D. O. TRINDADE, "Mensuração e Avaliação da Capacidade Inovativa de Micro, Pequenas e Médias Empresas: aplicação de métodos multicritério fuzzy de apoio à decisão," Mestre em Metrologia, PósMQI, Pontifícia Universidade Católica do Rio de Janeiro, Rio de Janeiro, 2016.

31. G. d. A. Martins, "Modelo Fuzzy AHP-TOPSIS para avaliação e seleção de tecnologias de geração de energia elétrica a partir de fontes renováveis," Mestre em Metrologia, PósMQI, PUC-Rio, Rio de Janeiro, 2017.

32. J. Hernández, Avaliação de impactos decorrentes da eficientização energética na indústria de papel e celulose utilizando modelo de decisão multicritério. Rio de Janeiro, 2018. 167p. Tese (Doutorado). Departamento de Engenharia Mecânica. Pontifícia Universidade Católica do Rio de Janeiro.

33. D. Štreimikienè, J. Šliogerienè, and Z. Turskis, "Multi-criteria analysis of electricity generation technologies in Lithuania," Renewable Energy, vol. 85, pp. 148-156, 2016.

34. H. Al Garni, A. Kassem, A. Awasthi, D. Komljenovic, and K. Al-Haddad, "A multicriteria decision making approach for evaluating renewable power generation sources in Saudi Arabia," Sustainable Energy Technologies and Assessments, vol. 16, pp. 137-150, 2016. 
35. S. E. Shmelev and J. C. Van Den Bergh, "Optimal diversity of renewable energy alternatives under multiple criteria: An application to the UK," Renewable and Sustainable Energy Reviews, vol. 60, pp. 679-691, 2016.

36. S. Hadian and K. Madani, "A system of systems approach to energy sustainability assessment: Are all renewables really green?," Ecological Indicators, vol. 52, pp. 194-206, 2015.

37. M. E. Arce, Á. Saavedra, J. L. Miguez, and E. Granada, "The use of grey-based methods in multicriteria decision analysis for the evaluation of sustainable energy systems: A review," Renewable and Sustainable Energy Reviews, vol. 47, pp. 924-932, 2015.

38. Ü. Şengül, M. Eren, S. E. Shiraz, V. Gezder, and A. B. Şengül, "Fuzzy TOPSIS method for ranking renewable energy supply systems in Turkey," Renewable energy, vol. 75, pp. 617-625, 2015.

39. M. Troldborg, S. Heslop, and R. L. Hough, "Assessing the sustainability of renewable energy technologies using multi-criteria analysis: Suitability of approach for national-scale assessments and associated uncertainties," Renewable and sustainable energy reviews, vol. 39, pp. 1173-1184, 2014.

40. E. Santoyo-Castelazo and A. Azapagic, "Sustainability assessment of energy systems: integrating environmental, economic and social aspects," Journal of Cleaner Production, vol. 80, pp. 119-138, 2014.

41. J. Mourmouris and C. Potolias, "A multi-criteria methodology for energy planning and developing renewable energy sources at a regional level: A case study Thassos, Greece," Energy Policy, vol. 52, pp. 522-530, 2013.

42. K. Baris and S. Kucukali, "Availibility of renewable energy sources in Turkey: Current situation, potential, government policies and the EU perspective," Energy Policy, vol. 42, pp. 377-391, 2012.

43. T. Kaya and C. Kahraman, "Multicriteria renewable energy planning using an integrated fuzzy VIKOR \& AHP methodology: The case of Istanbul," Energy, vol. 35, no. 6, pp. 2517-2527, 2010. 